\title{
¿Satisfecho con el Aprendizaje Basado en Proyectos?
}

Inmaculada Romero ${ }^{a}$, María Pachés ${ }^{a}$, Carmen Hernández-Crespo ${ }^{a}$ y $M^{\mathbf{a}}$ Teresa Sebastiá-Frasquet $^{\mathrm{b}}$

a Escuela Técnica Superior de Ingeniería de Caminos, Canales y Puertos (Universitat Politècnica de València) inrogi@dihma.upv.es, $\mathrm{y}^{\mathrm{b}}$ Escuela Politécnica Superior de Gandia (Universitat Politècnica de València)

\section{\$EWWDWW}

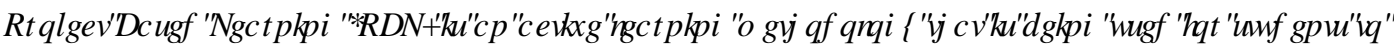

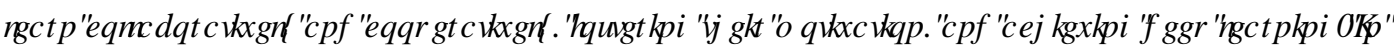

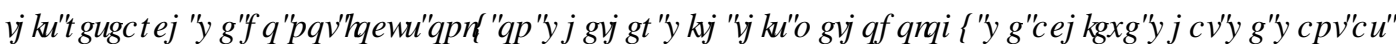

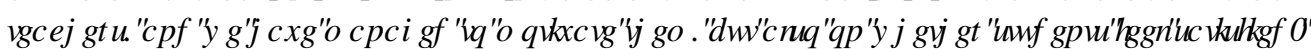

7KLWP HKRGRQ

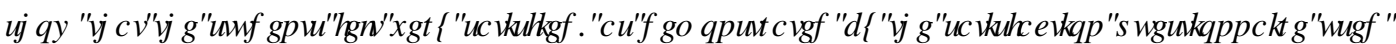

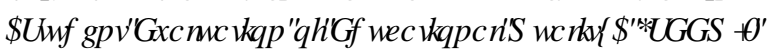

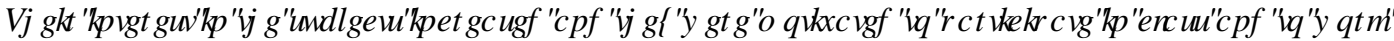

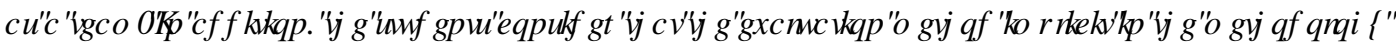

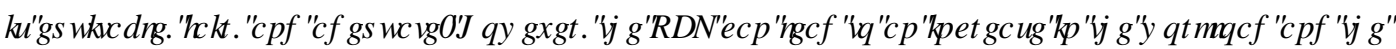

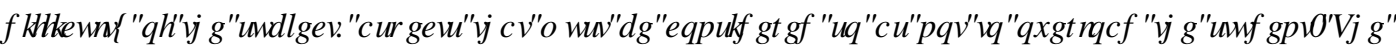

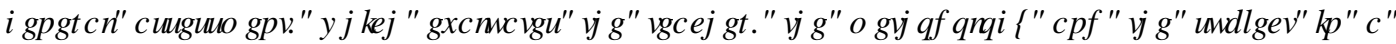
FRP SUHНHQYHP DQQHULИН WDRLGQОUT

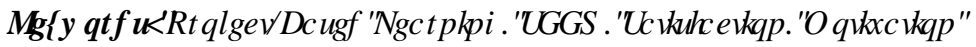

]

\section{HXP HQ}

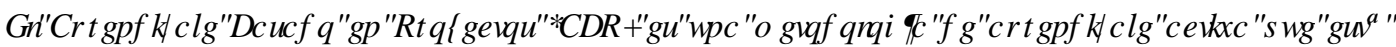

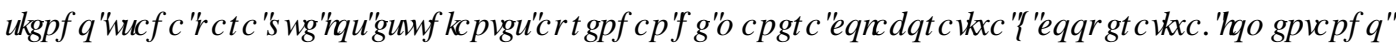

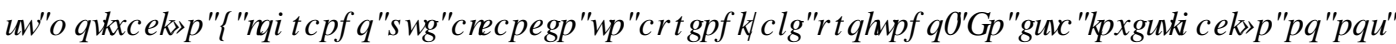

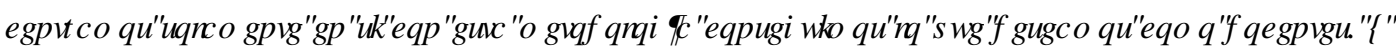

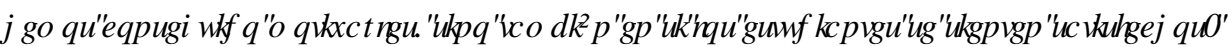

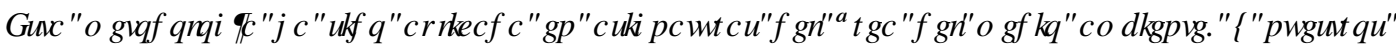

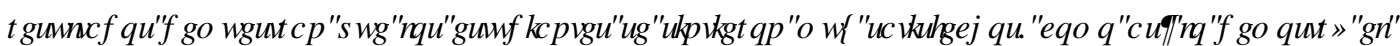

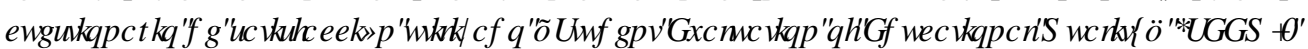

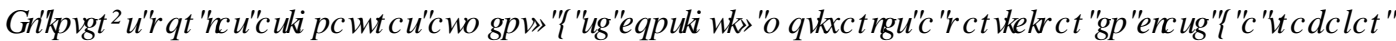

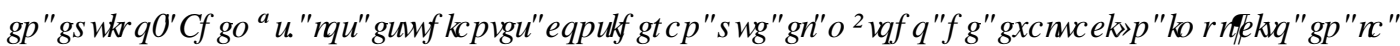

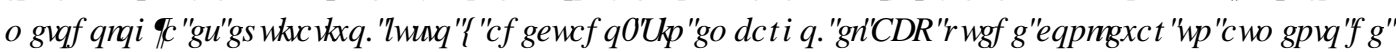

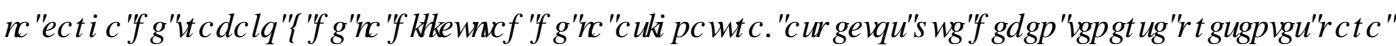

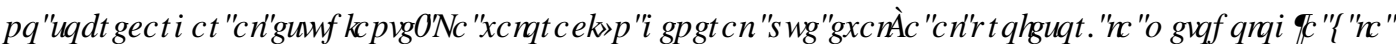

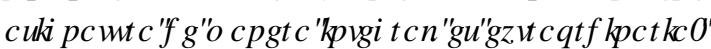

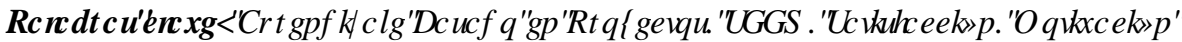




\section{Introducción}

El aprendizaje es el resultado de la interrelación de tres elementos, intención o motivo del estudiante, proceso o estrategia que utiliza y logros o rendimiento que obtiene (López-Aguado y López-Alonso, 2013). Es el denominado modelo 3P (Presagio-Proceso-Producto) de aprendizaje de Biggs (Sarzoza, 2013). Como explica López-Aguado y López-Alonso (2013), las variables presagio son previas al inicio del aprendizaje y se componen del perfil del estudiante y del contexto (variables personales, situacionales o contextuales). En cambio, las variables proceso se refieren al proceso del aprendizaje y está conformado por los motivos y estrategias, que se unen para formar los denominados enfoques de aprendizaje. Finalmente, las variables producto se relacionan con el resultado del aprendizaje y del rendimiento académico.

Cuando el sujeto enfoca su aprendizaje de manera superficial, estudia sin reflexionar sobre su objetivo o propósito final, sólo se centra en memorizar conocimientos y datos y en entrenar procedimientos de forma rutinaria. Su intención generalmente es afrontar simplemente los requerimientos del curso. Por ello suele encontrar dificultades para encontrar el sentido a los conocimientos nuevos, ve poco valor y sentido al curso y suele sentirse presionado y preocupado por el trabajo. Sin embargo, un sujeto que enfoca su aprendizaje de manera profunda consigue relacionar las ideas con la experiencia y el conocimiento previos, busca nuevos patrones, comprueba evidencias y las relaciona, y usa la memorización sólo cuando es necesaria. Por ello suele ser consciente de su propia comprensión y se interesa cada vez más de forma activa en el curso. El aprendizaje profundo se alcanza cuando el estudiante es capaz de encajar y conectar la nueva información con la previa, mediante relaciones de jerarquía, comparaciones o relaciones causa-efecto (Fasce, 2007; Fernández y Arquero, 2011; Hattie, 2013).

En algunas ocasiones se habla también del enfoque estratégico o de logro (Fasce, 2007; Fernández y Arquero, 2011), en el cual el sujeto utiliza una tercera manera de aprendizaje, la estrategia. El estudiante tiene la motivación de sacar buenas notas y aprobar, y busca la mejor y más organizada manera de conseguirlo. Como indica Fasce (2007) no es más que una estrategia superficial muy bien organizada. Aunque Fernández y Arquero (2011) apuntan que esta estrategia de lograr el éxito académico (no el aprendizaje por sí mismo) consiste en utilizar el enfoque superficial o profundo en función de los objetivos y de los métodos de evaluación.

Se ha visto que la estrategia de enseñanza que se emplee orientará el aprendizaje de diferente manera y por lo tanto los aprendizajes resultantes también diferirán (Fernández y Arquero, 2011; Díaz-Mujica y PérezVillalobos, 2013; López-Aguado y Gutiérrez-Provecho, 2014; Antonelli, 2017).

Un enfoque no es una característica del individuo, no es algo que el estudiante tiene. Como plantea Behar (2007), el enfoque describe una relación entre el estudiante y el aprendizaje que realiza. Así, cuando se plantea cambiar los enfoques no se pretende cambiar a los estudiantes, sino cambiar sus experiencias, sus percepciones, o sus concepciones de algo. Cada estudiante utilizará el enfoque superficial o el profundo como respuesta a diferentes circunstancias o tareas. Por lo tanto, conocer el enfoque de aprendizaje y los factores asociados, permitirá orientar mejor las estrategias de enseñanza y la manera de generar las condiciones apropiadas, para favorecer el aprendizaje profundo. Como apunta Monroy (2013), existen diferentes factores que influyen en los enfoques de aprendizaje: creencias epistemológicas, factores personales, factores contextuales (tipo de contenido o tarea, cantidad de contenidos, métodos de enseñanza, evaluación, etc.), la titulación académica, etc.

Los resultados obtenidos por Díaz-Mujica y Pérez-Villalobos (2013) muestran la existencia de relaciones directas entre la eficacia autorregulatoria (confianza de los estudiantes en el dominio y control de sus propios procesos de aprendizaje), la adopción de un enfoque profundo de aprendizaje y la utilización de estrategias de aprendizaje. 
Generalmente, un enfoque de aprendizaje superficial se relaciona con una falta de motivación a aprender, lo que a su vez da lugar a una disminución de la motivación. Sin embargo, un enfoque profundo se relaciona con sujetos altamente motivados, que genera en muchas ocasiones un aumento de la motivación (DíazMujica y Pérez-Villalobos, 2013). La estrategia profunda suele establecerse en base a una motivación intrínseca, orientada a querer saber, mientras que en la estrategia superficial la motivación suele ser externa, orientada a aprobar (Fasce, 2007).

Una de las metodologías que pueden usarse para favorecer la motivación del estudiante y el aprendizaje profundo es el Aprendizaje Basado en Proyectos (ABP). Con esta metodología, los estudiantes trabajan de manera activa, plantean el proyecto, trabajan de manera colaborativa, toman decisiones, lo implementan y lo evalúan. Una de sus características principales es que está orientado a la acción, es una herramienta que se centra en el estudiante, promoviendo su motivación. Además, estimula el aprendizaje colaborativo y cooperativo. Generalmente muchos de los Proyectos que se plantean se conciben como la búsqueda de una solución al planteamiento de un caso concreto del mundo real, como un problema ambiental o social. Se ha podido comprobar que el ABP aumenta la motivación de los estudiantes, pues el proceso comienza de manera que ellos mismos seleccionan los temas que más les interesen (Castro et al., 2018; Maldonado, 2008; Rodríguez-Sandoval et al., 2010; Katz y Chard, 1989). Durante el proceso, los estudiantes trabajan de manera activa y colaborativa, tomando decisiones continuamente. Se centra en el estudiante y promueve la motivación intrínseca (Martí et al., 2009), estimulando el aprendizaje colaborativo y cooperativo.

Para conocer el grado de satisfacción de los estudiantes con el empleo del ABP es necesario realizar una evaluación que aporte información del proceso y que permita reorientar el planteamiento inicial. Para ello los cuestionarios son una herramienta robusta, verificada y reconocida internacionalmente. El cuestionario SEEQ (Students' Evaluation of Educational Quality), es un instrumento ideal para la evaluación formativa puesto que permite analizar la eficiencia de la enseñanza (Andrade-Abarca et al., 2018; Moreira y Santos, 2016). Es una encuesta robusta, verificada y reconocida internacionalmente. Sus características psicométricas son elevadas (fiabilidad, validez, consistencia interna, etc.) (Marsh, 1982). Proporciona información sobre categorías diferentes, aprendizaje, entusiasmo, organización, interacción con el grupo, evaluación, carga de trabajo y dificultad y valoración general. Posee una última categoría de preguntas abiertas, con el objetivo de que los estudiantes puedan manifestarse abiertamente y aportar aquellos datos que crean que no encuentran recogidos en las preguntas precedentes.

Las asignaturas que son objeto de esta investigación están todas ellas relacionadas con el medio ambiente, y forman parte de titulaciones de ámbito técnico e ingenieril de la Universitat Politècnica de València:

- Máster de Ingeniería Ambiental. Escuela Técnica Superior de Ingeniería de Caminos, Canales y Puertos: Evaluación de Impacto ambiental; Calidad y Contaminación de Aguas

- Máster Universitario en Ingeniería Hidráulica y Medio Ambiente. Escuela Técnica Superior de Ingeniería de Caminos, Canales y Puertos: Modelación de la Calidad del Agua en el Medio Natural; Modelos de Calidad de Aguas Superficiales; Modelos de Calidad de Aguas Superficiales en la Gestión de Cuencas

- Grado de Ingeniería de Obras Públicas. Escuela Técnica Superior de Ingeniería de Caminos, Canales y Puertos: Ciencia e Impacto Ambiental de la Ingeniería Civil; Evaluación de Impacto Ambiental de la Ingeniería Civil; Medio Ambiente y Procesos Contaminantes

- Grado en Ciencias Ambientales. Escuela Politécnica Superior de Gandía: Evaluación y Gestión Ambiental; Groundwater management

A lo largo de los años se ha podido comprobar que algunos de los/las estudiantes que cursan estas asignaturas suelen adquirir aprendizajes superficiales y muy fragmentados. Esto es debido en parte a la poca transversalidad que tienen estos contenidos a lo largo de los cursos y a las metodologías de aprendizaje

(c)) BY-NC-ND 2021, Universitat Politècnica de València

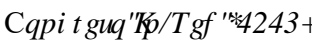


empleadas. Por ejemplo, los trabajos en equipo suelen terminar siendo una suma de trabajos individuales. A todo esto, hay que sumarle la poca motivación que tienen los/las estudiantes de las titulaciones más ingenieriles en alguna de las asignaturas por la falta de conciencia de la afección ambiental que sus competencias como futuros ingenieros tienen sobre el medio ambiente.

\section{Objetivos}

Nuestro objetivo general como docentes es mejorar la calidad del aprendizaje de los/las estudiantes, conseguir que alcancen un aprendizaje profundo que favorezca la transferencia de los conocimientos adquiridos en las diferentes asignaturas a su futura vida profesional y social. Pero además se desea que el estudiante se encuentre motivado en el proyecto y satisfecho con la práctica docente. Así, en este artículo se pretende comprobar la satisfacción de los estudiantes con la aplicación de esta metodología. Se plantean, por tanto, los objetivos específicos siguientes: Implementar el ABP en las distintas asignaturas, valorar si se ha logrado un mayor interés y motivación del estudiante en las asignaturas y evaluar si se ha conseguido aumentar la satisfacción del estudiante.

\section{Desarrollo de la innovación}

En esta investigación hemos implantado la metodología de ABP en seis asignaturas de ámbito ambiental en titulaciones de marco técnico e ingenieril, donde generalmente algunos estudiantes se muestran reacios de antemano a considerar que sus futuras obras de ingeniería podrían afectar al medio ambiente. Los estudiantes con los que trabajamos suelen mostrar poca disposición y suelen estar poco motivados desde el punto de vista ambiental, por lo que suele ser bastante difícil que consigan aprendizajes profundos.

La muestra coincide con la población total de estudiantes que asisten a las asignaturas, 124 estudiantes. Se propone un problema real a un grupo de estudiantes, que deben alcanzar una solución. Esta metodología les implica trabajar de forma colaborativa en un proyecto que deben diseñar, siguiendo unas pautas iniciales marcadas por el/la profesor/a, y donde cada estudiante tiene un rol individualizado con unos objetivos a alcanzar. Aunque el/la profesor/a supervisa continuamente el estado del proyecto, los/las estudiantes trabajan con total autonomía. Para ello, los pasos a seguir en cada asignatura fueron: definición del punto de partida o tema principal; formación de equipos colaborativos; definición del producto a desarrollar; organización y planificación (asignación de roles y definición de tareas y tiempos); búsqueda y recopilación de información; análisis y síntesis; realización de los proyectos; presentación del proyecto a otros compañeros.

Para valorar la satisfacción de los/las estudiantes, se utilizó el cuestionario SEEQ adaptado, formado por 27 ítems de escala Likert, con 5 opciones de respuesta. Este cuestionario evalúa 7 factores o dimensiones del proceso: Aprendizaje, Entusiasmo, Organización, Interacción con el grupo, Evaluación, Carga de trabajo y dificultad, y Otras Opiniones sobre la materia. Además, se incluyen tres preguntas abiertas que permiten que el estudiante pueda expresar lo que más le ha gustado, lo que debería mejorarse y cualquier otra opinión.

El esquema metodológico que se siguió fue aplicar el ABP en todos los grupos, y el último día de clase se pidió a los estudiantes que contestaran al cuestionario de satisfacción SEEQ. Los resultados obtenidos se analizaron mediante el paquete estadístico Statgraphics XVIII Centurion.

\section{Resultados}

Una vez recopilados todos los cuestionarios SEEQ se comprobó la normalidad de las variables mediante la prueba de Kolmogorov-Smirnov (KS). Se determinó la consistencia del cuestionario, obteniendo un alfa de Cronbach (estandarizada) de 0,813237 para las 7 variables (Límite inferior de confianza del 95\% Banda 
inferior de confianza $=0,771847$ ). Como regla general, un alfa igual a 0,7 o mayor se considera que representa un conjunto de variables consistente. A continuación, se muestran los resultados obtenidos para cada uno de los factores que considera el cuestionario tras su análisis.

El factor Aprendizaje (4 ítems) evalúa el interés de los/las estudiantes en la asignatura y si encontraron las clases estimulantes. Tal y como se observa en la Figura 1, la gran mayoría encontraron las asignaturas atractivas y estimulantes, aprendiendo cosas que consideran valiosas. Además, su interés por la materia aumentó y consideraron que aprendieron y comprendieron los contenidos de las asignaturas.

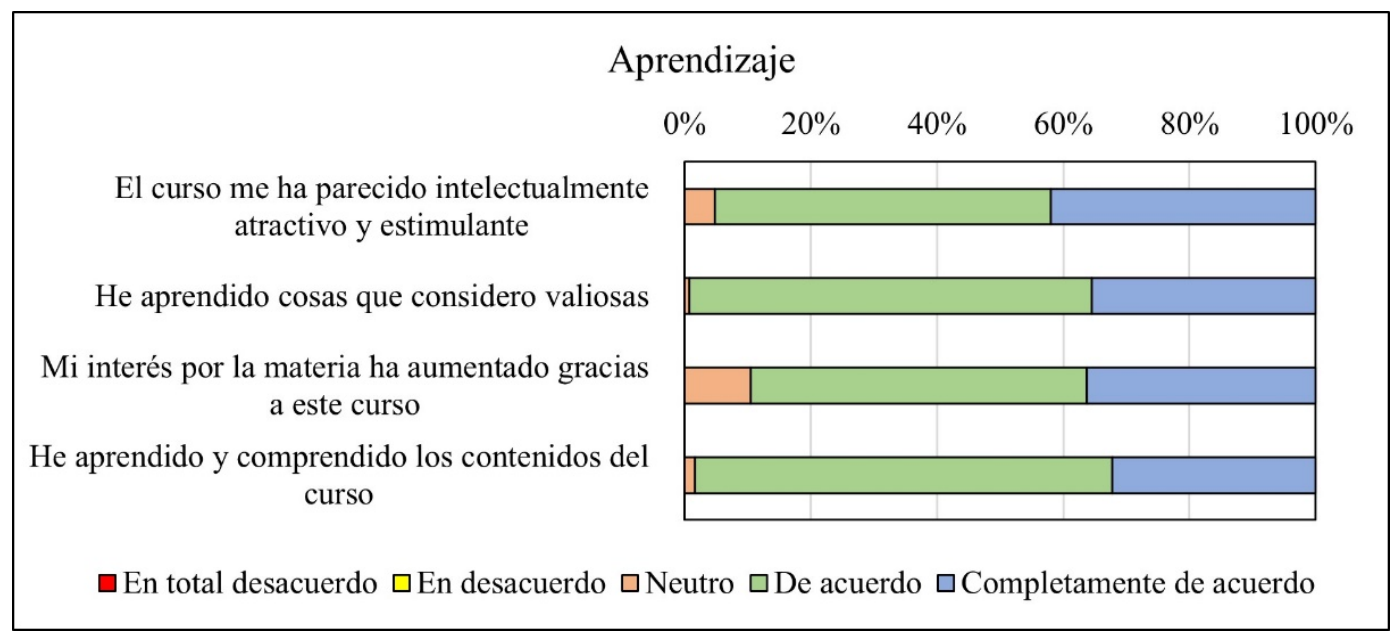

) $L \amalg \square S S U Q Q] D N A T$

El factor Entusiasmo (4 ítems) evalúa la actitud de la profesora y su habilidad para atraer la atención de los/las estudiantes con la pedagogía usada en clase. Generalmente el entusiasmo que el docente muestra en sus clases repercute positivamente en el interés de los estudiantes por la materia. Aquellos profesores que utilizan de manera adecuada el sentido del humor suelen tener a sus estudiantes más atentos e interesados. En nuestro caso así ha ocurrido, como se puede observar en la figura 2. Los resultados obtenidos son altamente satisfactorios, ya que más del $95 \%$ de los/las estudiantes consideran que las docentes han sido entusiastas y dinámicas y han conseguido mantener su atención en el aula.

El siguiente factor valorado es la Organización (4 ítems), que mide si el docente alinea los objetivos con los materiales de la asignatura y si están preparados y presentados apropiadamente. Generalmente, para que el aprendizaje sea efectivo es fundamental que el/la profesor/a haya planificado de manera correcta las actividades y tareas que se deben realizar. Es muy importante que se establezcan conexiones entre conceptos nuevos y ya aprendidos. Los resultados obtenidos (figura 3) demuestran que así ha sido. Aproximadamente el $80 \%$ de los/las estudiantes consideran que los objetivos de las asignaturas y las explicaciones eran claras, y que los materiales del curso estaban bien preparados.

Cuando se desea que los estudiantes trabajen en grupo, como es el caso de la metodología ABP aplicada en estas asignaturas, es necesario en muchas ocasiones motivarles a que lo hagan, es decir, fomentar la participación. Es lo que evalúa el factor Interacción con el grupo (6 ítems), en el que se valora la habilidad de la profesora para motivar a los estudiantes a participar de discusiones en clase (figura 4). Las docentes han conseguido motivar a casi el $95 \%$ de los estudiantes a participar en clase y a trabajar en equipo. Sin embargo, un pequeño porcentaje de los estudiantes (3\%) consideran que la interacción con sus compañeros de equipo no ha sido del todo satisfactoria. Incluso un $2 \%$ de los estudiantes indican que no todos los miembros del grupo han contribuido activamente. La experiencia demuestra que, en todas aquellas metodologías de aprendizaje que requieren el trabajo en grupo, puede existir el alumno "estratega", que se beneficia del grupo sin aportar nada. La existencia de conflictos en los trabajos en equipo es bastante 
habitual, pudiendo deberse a conflictos por las tareas o por las relaciones (Benítez et al., 2011). Los primeros son debidos a los desacuerdos que puede haber entre los estudiantes sobre el contenido concreto de la tarea o la forma de llevarla a cabo. Los conflictos por relaciones se deben fundamentalmente a incompatibilidades entre los miembros del equipo. En cada caso concreto, las razones pueden ser diversas, desde la formación del equipo (voluntaria, aleatoria, pseudoaleatoria, decidida por el docente...), la falta de comunicación asertiva entre compañeros, la existencia de un estudiante aventajado, o incluso la existencia en el equipo de algún "jeta" o algún "manta" (Del Canto et al., 2009; Navarro y Valero, 2008). Para evitar o disminuir los conflictos, el docente debe ser un orientador y guía que, junto a la labor grupal, conseguirá motivar un aprendizaje cooperativo y colaborativo, generando por tanto un razonamiento crítico y reflexivo (Benítez et al., 2011; Viceconte, 2015).

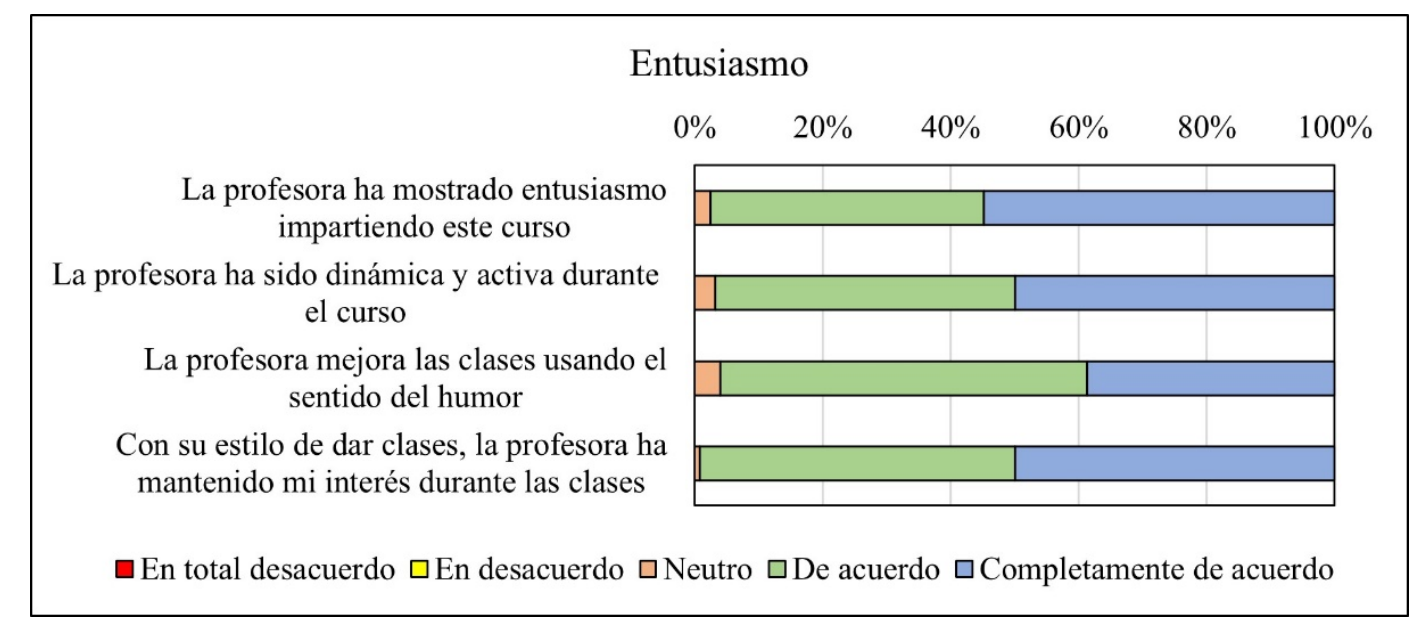

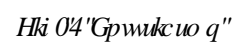

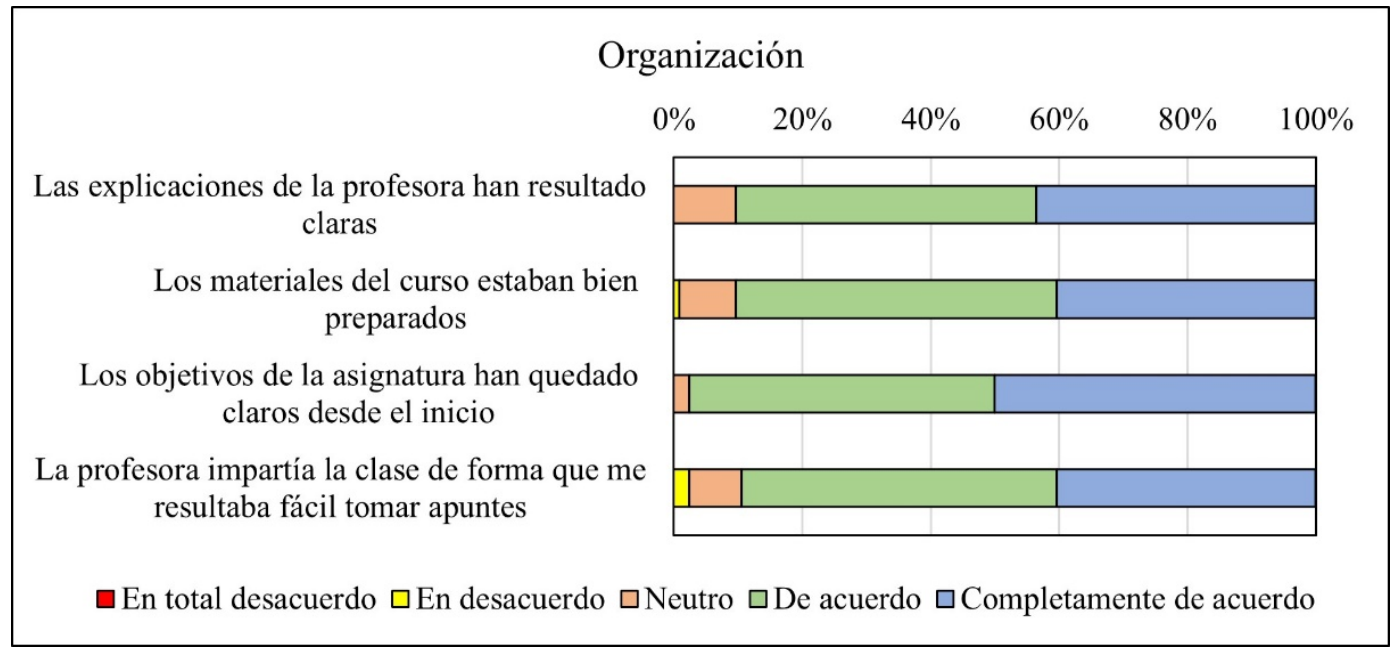

) $L$ W[2 UDQT]DFIQ 


\section{Interacción con el Grupo}

Se animaba a los estudiantes a participar en las discusiones de clase

Se animaba a los estudiantes a compartir sus ideas y conocimientos

Se animaba a los estudiantes a preguntar y se les daba respuestas satisfactorias

Se animaba a los estudiantes a expresar sus ideas y a cuestionar las expresadas por el profesor

Durante la realización de la actividades en grupo, la interacción con el resto de miembros del equipo ha sido satisfactoria

Para la realización de los informe de prácticas, todos los miembros del grupo han contribuido activamente

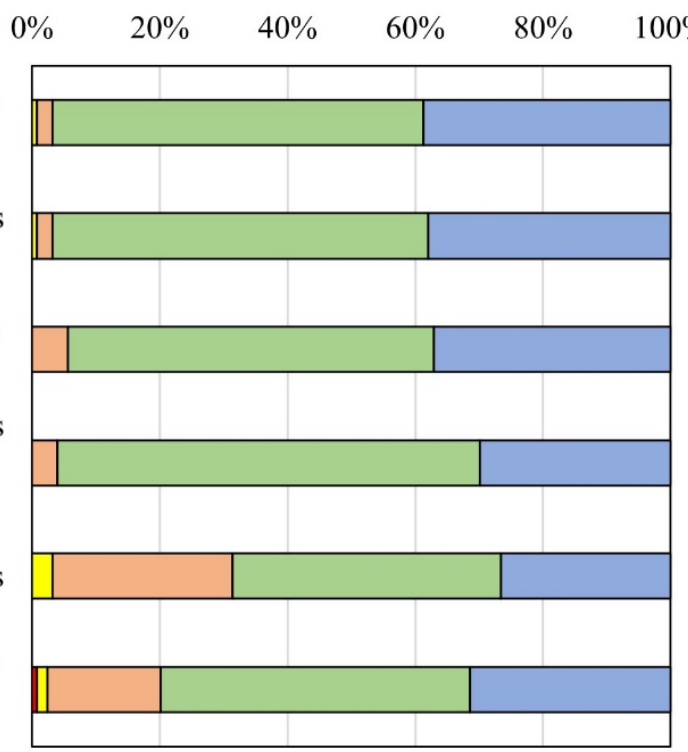

$\square$ En total desacuerdo $\square$ En desacuerdo $\square$ Neutro $\square$ De acuerdo $\square$ Completamente de acuerdo

\section{) $L \amalg \square, Q U A D F F Y$ QFRQHDJ LXSR}

El factor Evaluación (2 ítems) mide si el método de evaluación se considera justo, apropiado y alineado con los contenidos. En la figura 5 se observan los resultados obtenidos. El 100\% de los/las estudiantes consideran que la evaluación utilizada se centra en los contenidos de las asignaturas. Además, el 95\% considera que los métodos de evaluación son equitativos, justos y adecuados.

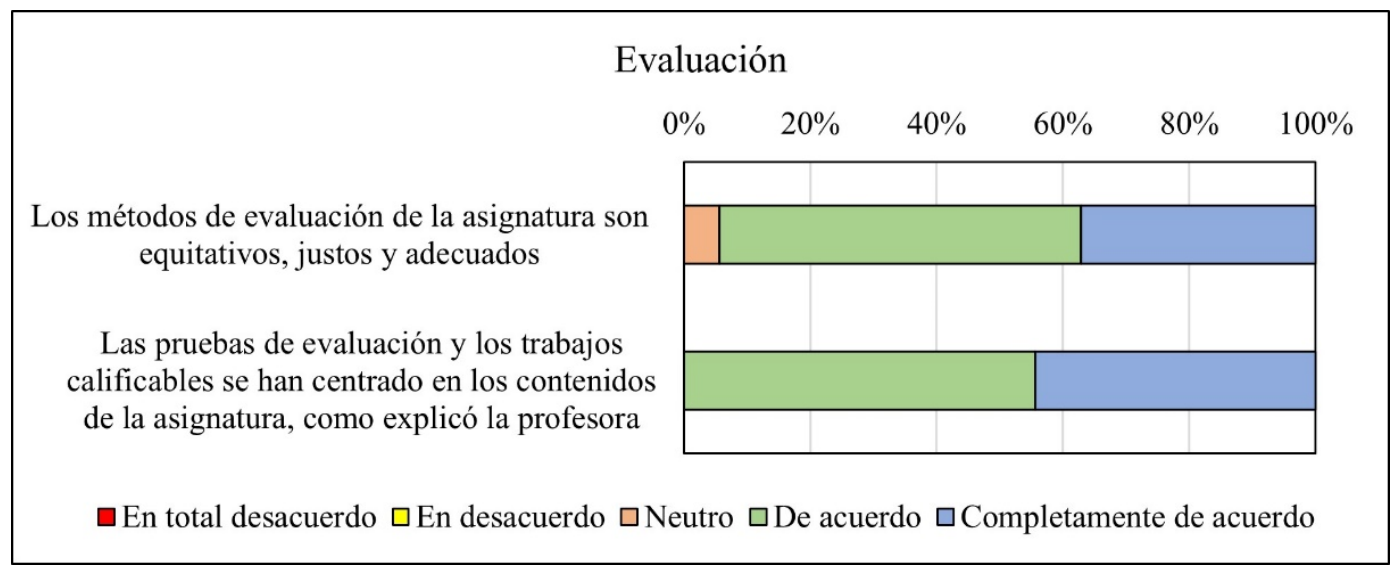

\section{) $L J \mathbb{W} \square($ YDODFlyQ}

En el factor Carga de trabajo y dificultad (4 ítems) se evalúa la percepción del estudiante respecto a la carga de trabajo que conlleva aplicar esta metodología y la dificultad de la asignatura respecto al resto de materias. Este factor es indispensable tenerlo presente para evitar cargar a los/las estudiantes con un exceso de trabajo. En la figura 6 se observa los resultados obtenidos, donde se debe tener presente que este factor es opuesto a los restantes, siendo los valores 4 y 5 los menos deseables. El 20\% de los/las estudiantes considera que la asignatura es más difícil que las restantes que cursan y un 35\% considera que la carga de trabajo es grande o muy grande. Un 38\% invierten en las actividades individuales más de 15 horas no presenciales y en las grupales más de 16 horas. 


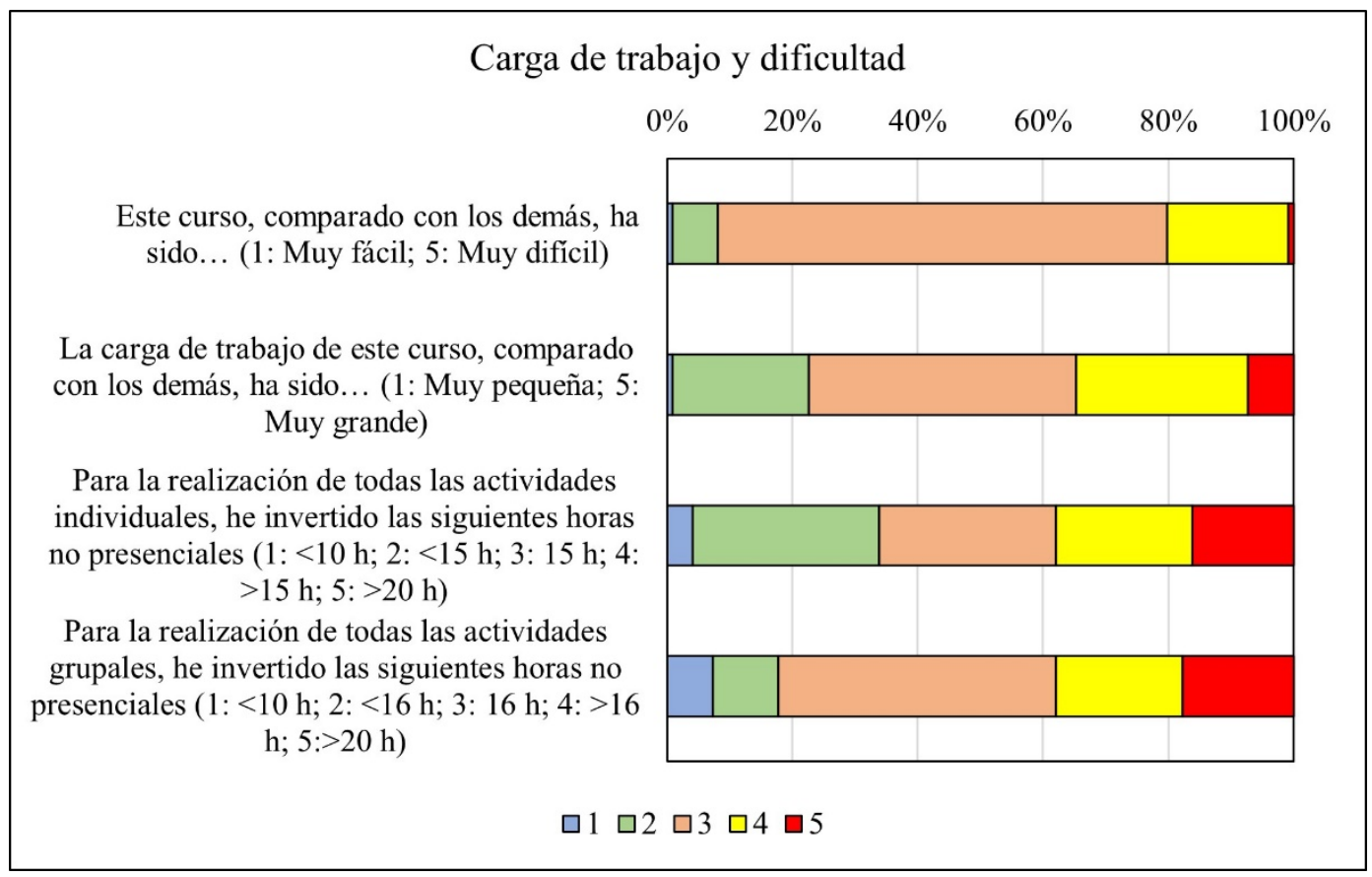

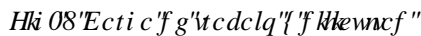

El último factor tiene en cuenta Otras opiniones ( 3 ítems), donde se recoge información sobre la valoración global de la asignatura. En la figura 7 se observa que casi el $48 \%$ de los/las estudiantes consideran que la asignatura es mejor que la mayoría de las que han cursado y el $50 \%$ que es igual. Sólo un $2 \%$ considera que es peor que otras. Respecto al nivel de interés que tenían en la asignatura antes de cursarla, un 11\% indican que su interés era reducido, pero un $55 \%$ que era elevado o muy elevado. Casi la totalidad de los/las estudiantes (un 99\%) esperaban aprobar la asignatura, siendo un $80 \%$ los que pensaban obtener como mínimo un 7 (notable) y casi un 20\% más de 9 (sobresaliente).

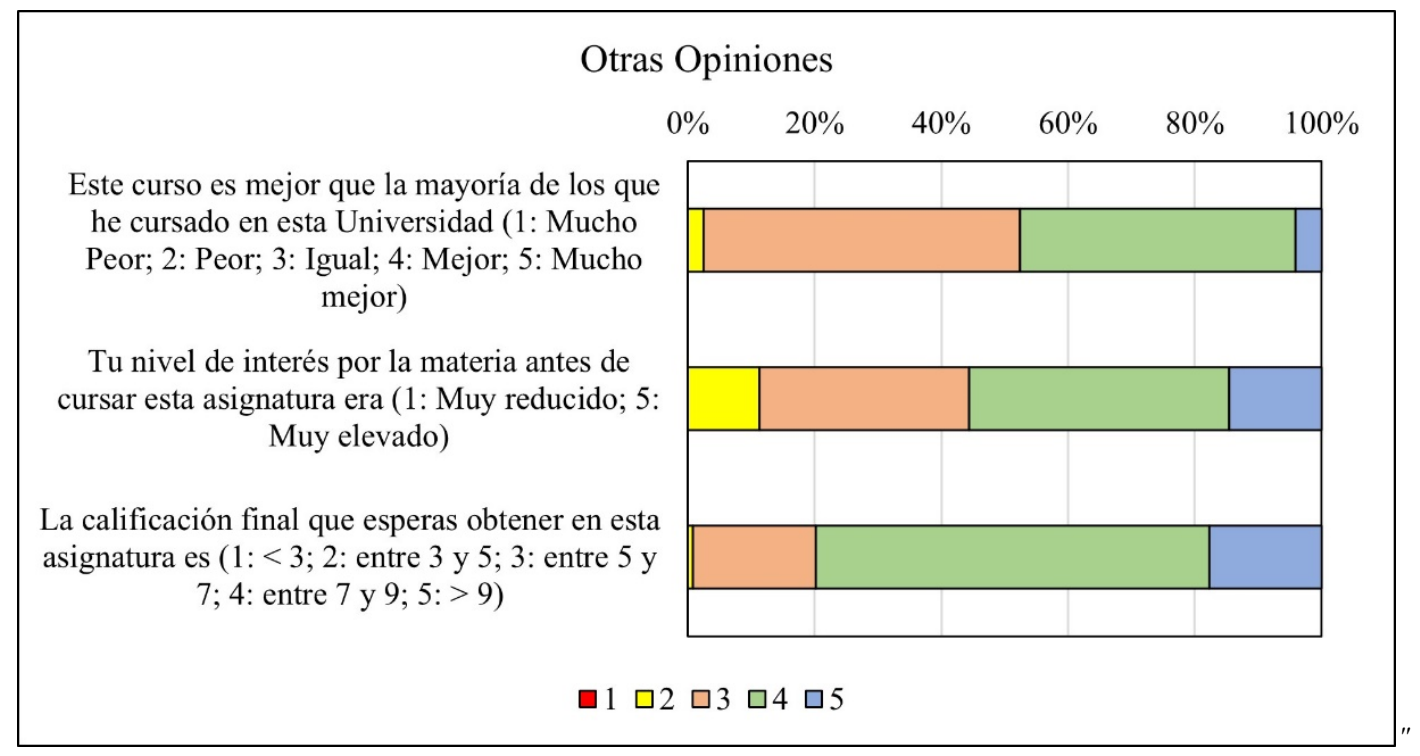

) $L \amalg \square 2$ WOWRSIQRQHW

Las 3 preguntas abiertas que se plantean en el cuestionario permiten que los/las estudiantes indiquen todos aquellos otros aspectos que consideran importantes y que no están recogidos en las preguntas anteriores. 
Entre todas las respuestas obtenidas se recoge a continuación una pequeña muestra respecto de las profesoras, de las asignaturas y de la metodología de ABP:

- Indica las características de esta asignatura que te han ayudado más en el proceso de aprendizaje:

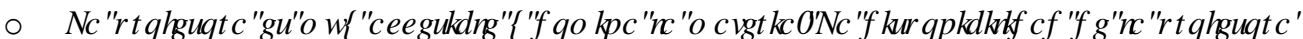

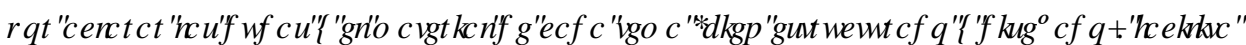
HODSULQ] DNFII

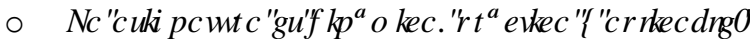

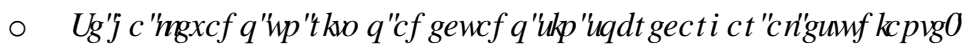

- Indica las características de esta asignatura que deberían mejorarse de manera prioritaria (especialmente, aspectos no considerados en las preguntas anteriores)

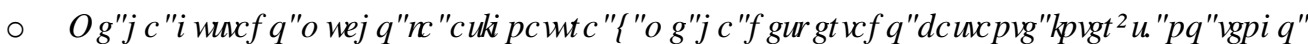
QQJ XQDREVHUDFIY QSDWP HRUTT

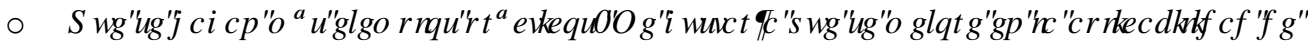
DDP DUAIDFRQP i VHNAFIFIRV

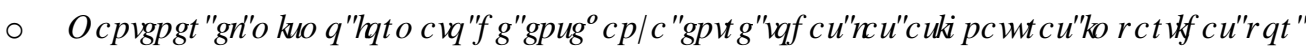
RWRVISLRIHRLHW

- Añade, si lo deseas, cualquier otro comentario personal sobre el desarrollo de la asignatura:

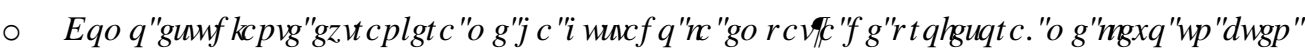

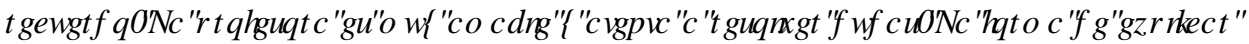

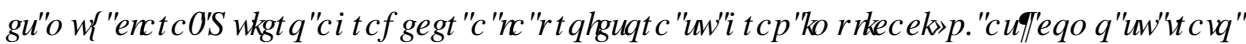

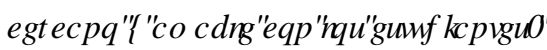

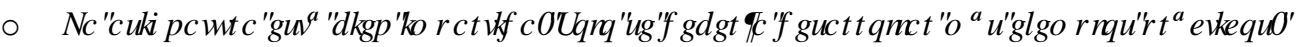

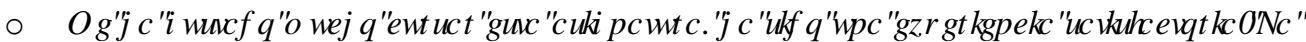

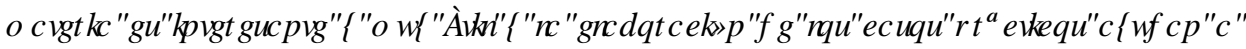

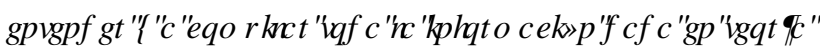

Todas las respuestas obtenidas en estas tres preguntas abiertas dejan patente el agradecimiento de los/las estudiantes respecto a las docentes, a las asignaturas y al ABP.

\section{Conclusiones}

Tras implementar el ABP en las distintas asignaturas, se ha conseguido un mayor interés y motivación del estudiante en las asignaturas y se ha aumentado su satisfacción. Por tanto, se considera una buena metodología para aquellas asignaturas en las que quizá el/la estudiante, a priori, no encuentra una clara utilidad dentro de su plan de estudios, como puede ser la formación ambiental en titulaciones ingenieriles.

Como resultado de la aplicación de la metodología, los/las estudiantes la consideran totalmente adecuada para las asignaturas cursadas, e incluso algunos indican que les gustaría que otras asignaturas también utilizaran el ABP. El interés por las asignaturas aumentó, fomentando, por tanto, un aprendizaje profundo, y se consiguió motivarles a participar en clase y a trabajar en equipo. Además, consideran que el método de evaluación implícito en la metodología es equitativo, justo y adecuado.

Sin embargo, el ABP puede conllevar un aumento de la carga de trabajo y de la dificultad de la asignatura, aspectos que deben tenerse presentes para no sobrecargar al estudiante. Así ha ocurrido en estas asignaturas, pues, aunque los/las estudiantes se encuentran satisfechos con la metodología, algunos indican que la carga de trabajo es grande o muy grande.

La aplicación del $\mathrm{ABP}$ ha conseguido que los/las estudiantes se encuentren muy satisfechos con la metodología aplicada. Han valorado muy positivamente que las docentes tengan una muy buena habilidad 
para relacionarse con ellos y ser accesibles antes, durante y después de las sesiones de aula y consideran además que son empáticas. La valoración general, que evalúa al profesor y la asignatura de manera integral, es extraordinaria.

Además, el uso de este tipo de cuestionarios de satisfacción consigue que los/las estudiantes reflexionen sobre su propio proceso de aprendizaje, afianzándolo, y, por tanto, contribuye a aumentar su madurez y el aprendizaje a lo largo de la vida.

\section{Agradecimientos}

El proyecto de innovación y mejora educativa en el que se enmarca esta comunicación ha recibido el apoyo económico del Instituto de Ciencias de la Educación (ICE) de la Universitat Politècnica de València (UPV) Proyecto de Innovación y Mejora Educativa (PIME/19-20/174), “Objetivo Agenda 2030 y UPV 2020: Aprendizaje ambiental profundo en la UPV".

\section{Referencias}

Andrade-Abarca, P.S.; RAmón-Jaramillo, L.N. y LoAizA-AguirRe, M.I. (2018). “Aplicación del SEEQ como instrumento para evaluar la actividad docente universitaria” en 5HLWDGH, QVHMWDFIy Q( GXFDUMD, vol. 36, issue 1, p. 259-275. <http://dx.doi.org/10.6018/ rie.36.1.260741> [Consulta: 15 de febrero de 2021]

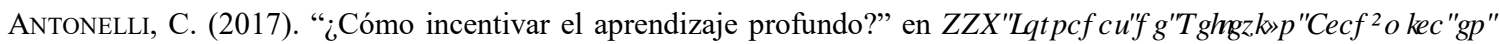
' LMKR]I L\&RP XQFDFly Q vol. 30, p. 133-135.

BEHAR, R. (2007) “¿Aprendizaje superficial o aprendizaje profundo? Discusión sobre los factores que intervienen” en + HXUMAFD, vol. 14, p. 67-76. <http://hdl.handle.net/10893/6117> [Consulta: 25 de febrero de 2021]

Benítez, M., Medina, F.J, y Munduate. L. (2011). "El estudio del conflicto en los equipos de trabajo. Una visión de las contribuciones científicas realizadas en España (Studying conflict in work teams. A review of the spanish scientific contribution)" en 3DSHOMLGDB UFy $\mathbb{R J} R$, vol. 32, issue 1, p. 69-81.

Castro, A., López, G., Padilla A.L., Meléndez, L. y Escobedo, A.B. (2018). "La investigación de un proyecto usado como estrategia para valorar el impacto ambiental por alumnos de química" en 5HILLD( OFFUy QIFD VREUH \&XHSRKS FDCPP IFRVI I* UXSRVGH, QYHWWDFIy Q vol. 5, issue 10, p. 1-14.

Del Canto, P., Gallego, I., López, J.M., Mora, J., Reyes, A., Rodríguez, E., Sanjeevan, K., Santamaría, E., y VALERO, M. (2009). "Conflictos en el trabajo en grupo: cuatro casos habituales" en 5 HILWDGH) RLP DFIy QH, QQRYDFIyQ ( GXFDUMD8 QYYHИWUD, vol. 2, issue 4, p. 211-226.

Díaz-Mujica, A. y PÉrez-Villalobos, M.V. (2013). "Autoeficacia, enfoque de aprendizaje profundo y estrategias de

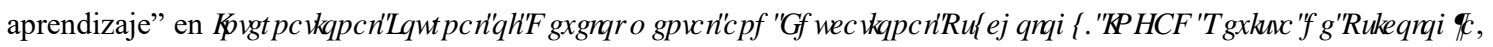
vol. 2, issue 1, p. 341-346.

FASCE, E. (2007). "Aprendizaje profundo y superficial, Tendencias y perspectivas" en 5HW( GXFL\&IHQFL6DXG vol. 4, issue 1, p. 7-8.

FernándeZ, C. y ARQuero, J.L. (2011). "Evaluación de innovaciones y enfoques de aprendizaje. Presentación preliminar de un instrumento de medida. Innovations assessment and approaches to learning. Preliminar presentation of a questionnaire" en ,95-RLQDCDUGH, QQRIDFIy QH, QYHMUDFIyQ' RFHQM Sevilla: Edicion Digital Atres. 214-225. $<$ http:/hdl.handle.net/11441/43202> [Consulta: 14 de febrero de 2021]

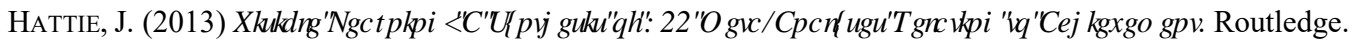

KATZ, L.G. y CHARD, S.C. (1989). ( QJDIQJ LFKTCHHQMPP IQGVI7 KH3 URNFFWDSSLRDFK. Norwood, NJ: Ablex.

LópeZ-Aguado, M. y GutiérReZ-Provecho, L. (2014). "Modelo explicativo del efecto de los enfoques de aprendizaje sobre el rendimiento y el papel modulador de la dedicación temporal" en 5HLWDLGH, QYHWWDFIy Q( GXFDMLD, vol. 32, issue 2, p. 447-462. <http://dx.doi.org/10.6018/rie.32.2.164761> [Consulta: 2 de diciembre de 2020] 
LÓPez-Aguado, M. y LóPeZ-Alonso, A.I. (2013). "Los enfoques de aprendizaje. Revisión conceptual y de investigación //Learning Approaches: Theoretical and Research Review" en 5HMLDL\&RQRP EIDQDGH( GXFDFlyQ vol. 64, p. 131-153.

MALDONADO, M. (2008). “Aprendizaje basado en proyectos colaborativos. Una experiencia en educación superior” en / DXUVV, vol. 14, issue 28, p. 158-180.

MARSH, H.W. (1982) “SEEQ: A reliable, valid, and useful instrument for collecting students' evaluations of university teaching”, en \%UMMK-RXLQDORIL GXFDMRQDOB XFKRQRJ।, vol. 52, p. 77-95.

Martí, J.A., Heydrich, M., Rojas, M. y Hernández, A. (2009). “Aprendizaje basado en proyectos: una experiencia de innovación docente” en 5 HLWD8 QHHWGDCF( \$) ,7, vol. 46, issue 158, p. 11-21.

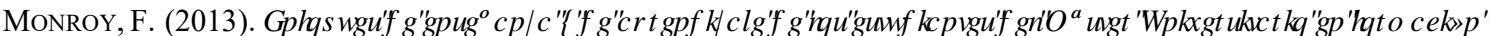
GH 3LRIHRLAR GH (GXFDFYQ 6HFQGDUD. Tesis doctoral. Universidad de Murcia. 319pp. $<$ http://hdl.handle.net/10201/31224> [Consulta: 13 de diciembre de 2020]

Moreira, L.M., y SANTOS, M.A. (2016). “Evaluando la enseñanza en la Educación Superior: percepciones de docentes y discentes. Evaluating Teaching in Higher Education: Teacher and Student Perceptions" en 5HLMDHDFWU QFDDCH

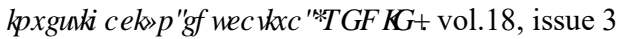

Navarro, J.J. y VAlero, M. (2008). “Cómo enfrentarse a jetas y mantas”, traducción de OAKLeY, B., FelDER, R.M., BRENT, R., y ELHAJJ, I. (2004). "Coping with hitchhikers and couch potatoes on teams" en - RXLQDORI6WOHQH\&HQHHHE / HDQQU, vol. 2, issue 1, p. 32-34.

Rodríguez-Sandoval, E., Vargas-Solano, E.M. y LunA-Cortés, J. (2010). "Evaluación de la estrategia aprendizaje basado en proyectos" en ( GXFDFYQI L( GXFDRRUN, vol. 13, issue 1, p. 13-25.

SARzOZA, S. (2013). “Aprendizaje desde la perspectiva del estudiante: Modelo teórico de enseñanza y aprendizaje 3P” en \$FFYQ Q HODJ JIFD, vol. 22, p. $114-121$.

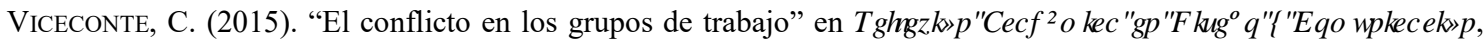
$\mathrm{N}^{\circ} \mathrm{XXV}$, vol. 25 , p. $85-87$. 